\title{
Multiple Types of Childhood Maltreatment, Sleep, and Anxiety in Former Foster Youth
}

\author{
Hyunji Lee ${ }^{1}$ D $\cdot$ Rachel A. Fusco ${ }^{2}$
}

Accepted: 28 January 2021

(c) The Author(s), under exclusive licence to Springer Science+Business Media, LLC part of Springer Nature 2021

\begin{abstract}
Although existing literature has widely identified significant relationships between a history of childhood maltreatment and mental health problems and between a history of childhood maltreatment and sleep problems, less is known about relationships between experiences of multiple types of childhood maltreatment, sleep problems, and anxiety in low-income young adults and former foster youth. This study aimed to explore whether experiences of multiple types of childhood maltreatment, a history of foster care placement, lifetime trauma, and hours of sleep would be associated with anxiety in low-income young adults and former foster youth. The study surveyed a total sample of 143 young adults, and their hours of sleep were measured with FitBits. Multiple regression analyses were conducted to examine the relationships between experiences of multiple types of childhood maltreatment, a history of foster care placement, lifetime trauma, hours of sleep, and anxiety. The study found a significant relationship between multiple types of child maltreatment and anxiety, after controlling for a history of foster care placement, lifetime trauma, and demographic information. Notably, while experiences of multiple types of child maltreatment and a history of foster care placement were no longer associated with anxiety, a lifetime history of trauma remained associated with anxiety, after controlling for hours of sleep. Social workers and service providers need to provide low-income young adults and former foster youth with interventions aimed at addressing both sleep problems and traumatic stress, which may in turn reduce the risk for anxiety.
\end{abstract}

Keywords Multiple types of childhood maltreatment $\cdot$ Sleep $\cdot$ Anxiety $\cdot$ Foster care $\cdot$ Young adults

The Children's Bureau's annual child maltreatment report for FFY 2018 showed that 678,000 children were reported to Child Protective Services (CPS) as victims of child abuse and neglect, and 146,709 victims received foster care postresponse services in 2018 (Department of Health \& Human Services; U.S. DHHS 2020). Of the total victims, $22.9 \%$ were separated from their families of origin and entered foster care in 2018 (U.S. DHHS 2020). It has been documented that foster children and youth (Pilowsky and $\mathrm{Wu}$ 2006) and foster youth transitioning to adulthood (Havlicek et al. 2013) have poorer mental health outcomes, as

Hyunji Lee

hyl34@pitt.edu

Rachel A. Fusco

rachel.fusco@uga.edu

1 School of Social Work, University of Pittsburgh, 4200 Fifth Ave, Pittsburgh, PA 15260, USA

2 School of Social Work, University of Georgia, 279 Williams Street, Athens, GA 30602, USA compared to non-foster children and youth. For example, a study using a nationally representative sample of adolescents indicated that foster youth had higher risk for anxiety and mood symptoms as well as substance use disorders than did youth without a history of foster care placement (Pilowsky and $\mathrm{Wu}$ 2006). In addition, Havlicek et al. (2013) showed that while the prevalence of mental health disorders was higher among foster youth transitioning to adulthood than was non-foster youth, the rate of mental health service utilization decreased as foster youth transitioned to adulthood. Moreover, Farmer et al. (2001) reported that mental health problems were prevalent not only among youth in foster care but among youth living in poverty who had never been in foster care, but the youth living in poverty were less likely to use mental health services. Notably, Farmer et al. (2001) found that youth living in poverty, especially those without public health insurance had lower rates of mental health service utilization, placing them at higher risk for mental health problems. Although there is evidence that childhood experiences of maltreatment are associated with an elevated risk 
for having low-income status and mental health problems in adulthood (Jones et al. 2018), research on the relationship between childhood experiences of multiple types of maltreatment and anxiety among low-income young adults has been understudied.

While an increasing body of research has reported a high prevalence of mental health problems in foster care populations and foster care alumni (Havlicek et al. 2013; Pilowsky and $\mathrm{Wu}$ 2006), few studies have examined why foster care populations are at greater risk for poor mental health outcomes (Hillen and Gafson 2015). Of the few studies, some studies have suggested that entering foster care per se would contribute to mental health problems because separation from their families of origin (Szilagyi 2012) or experiences of multiple placements could be a stressor (Bass et al. 2004; Hillen and Gafson 2015; Rubin et al. 2007). Alternatively, other studies have identified that childhood experiences of maltreatment significantly predict mental health problems among foster care populations (Lawrence et al. 2006; Swire and Kavaler 1977). One recent study also noted that foster youth, especially those transitioning to adulthood are at higher risk for mental health problems because they face multiple challenges regarding independent living, such as economic hardships (Häggman-Laitila et al. 2019).

Since prior research has not widely investigated mechanisms by which childhood experiences of maltreatment lead to mental health problems in former foster youth and low-income young adults, it is important to identify specific factors (e.g., multiple types of child maltreatment, sleep problems, etc.) that could better explain the high prevalence of mental health problems. Specifically, there is research evidence that multiple types of childhood maltreatment are associated with high levels of depression in youth aging out of foster care (Salazar et al. 2011) and that sleep problems are prevalent among those with a history of childhood maltreatment (Glod et al. 1997; Greenfield et al. 2011).

Given the high prevalence of mental health problems and sleep problems among those with a history of childhood maltreatment, it is critical for the survivors of child maltreatment to receive interventions aimed at addressing both mental health problems and sleep problems. Since sleep-focused interventions have been evidenced as effective for reducing stigma in the use of mental health services as well as mental health problems (Ho et al. 2016), interventions targeting sleep problems can be used to treat mental health problems in former foster youth and low-income young adults. To the best of our knowledge, there is no prior research examining associations between experiences of multiple types of childhood maltreatment, sleep problems, and anxiety in former foster youth and low-income young adults who are at high risk of mental health problems. Thus, while focusing on sleep problems, the current study explored the relationships between multiple types of child maltreatment and anxiety in order to gain a better understanding of the high prevalence of mental health problems. The results of the study could also guide social workers or service providers to develop and implement more effective interventions for treating anxiety.

\section{Mental Health Problems in Former Foster Youth}

Existing literature has documented detrimental effects of a history of childhood maltreatment on a range of mental health problems among foster children and youth (Lawrence et al. 2006; Swire and Kavaler 1977). It has been argued that the process of placement in foster care can be a stressor that disrupts the development of mental health in foster children and youth (Bass et al. 2004). Specifically, Bass et al. (2004) reported that placement instability in foster care, such as multiple placements, could lead to poor health outcomes. Some studies have also shown that youth and young adults aging out of foster care particularly face more challenges while transitioning to adulthood (Courtney et al. 2011; Häggman-Laitila et al. 2019). For example, a systematic review of 13 studies of the transition experiences among former foster youth reported that those transitioning to adulthood had a lack of resources for stable housing, education, and employment, all of which could interrupt their independent living (Häggman-Laitila et al. 2019). The study also revealed that those in the transition had adverse mental health outcomes, which might be attributed to the multiple stressors regarding independent living (Häggman-Laitila et al. 2019).

In addition, another body of literature has documented that trauma histories, particularly child maltreatment, are associated with an increased risk for poor mental health outcomes in both current foster youth and former foster youth (McMillen et al. 2005; Pecora et al. 2009; Shin 2005). For example, a review of 32 studies identified that foster children and youth had higher prevalence rates of mental health disorders (e.g., anxiety, depression, etc.) and developmental problems (Oswald et al. 2009). Similarly, another review study examining mental health concerns in current foster youth and former foster youth indicated that those with trauma histories exhibited severe mental health problems, such as PTSD, depression, and anxiety (Pecora et al. 2009). Moreover, young adults who aged out foster care had higher rates of PTSD symptoms, depression, anxiety, substance use, and other mental health disorders, compared to their counterparts from the general population (Pecora et al. 2009).

While it has been reported that foster care populations are more likely to use mental health services than the general populations (Farmer et al. 2001), some studies have pointed out that youth aging out of foster care are less likely to utilize mental health services (Havlicek et al. 2013; Villagrana et al. 2018). Specifically, Farmer et al. (2001) found 
that youth in foster care were more likely to use mental health services, whereas Shin (2005) reported that foster care alumni were less likely to use mental health services. Shin (2005) explained that social stigma in the utilization of mental health services could discourage youth in foster care from continuing to use mental health services. Villagrana and colleagues (2018) also stated that stigma associated with mental health service utilization appeared to hinder the use of mental health services among foster care alumni.

Although there is research evidence that Cognitive Behavioral Therapy (CBT) is effective for alleviating posttraumatic stress symptoms among foster care populations (Ai, Foster, Pecora, Delaney and Rodriguez 2013) and that sleep-focused CBT reduces anxiety symptoms (Pillai et al. 2015) and posttraumatic stress (Ho et al. 2016), it appears that there should be another intervention that can better address stigma in the use of mental health services and increase accessing mental health services for foster care alumni and low-income young adults. Specifically, computerized CBT, which delivers services via internet, has been demonstrated as effective for mitigating insomnia (Cheng and Dizon 2012), posttraumatic stress (Ivarsson et al. 2014), and anxiety and depressive symptoms (Andrews et al. 2010). Since computerized CBT and sleep-focused CBT can contribute to reducing stigma in the use of face-to-face CBT, the sleep-focused CBT delivered over the internet can benefit foster care alumni and low-income young adults who are at high risk for mental health problems and sleep problems.

\section{Childhood Maltreatment and Sleep Problems}

Prior research has identified adverse childhood experiences (ACEs) as being significantly associated with sleep problems (Brindle et al. 2018; Glod et al. 1997; Greenfield et al. 2011; Laskemoen et al. 2020). Specifically, sleep problems were prevalent among children who were physically abused than were children who were not abused (Glod et al. 1997). In addition, another study identified that childhood experiences of traumatic events, including general disaster, were related to an increased risk for poor sleep quality in adulthood (Brindle et al. 2018). Moreover, using data from a nationally representative sample of adults, one study revealed that frequent experiences of childhood maltreatment were associated with poor sleep quality in adulthood (Greenfield et al. 2011). In line with this, a more recent study of a clinical sample of adults reported that the prevalence of sleep problems, especially insomnia, was significantly higher in those with a history of childhood maltreatment, compared to those without a history of childhood maltreatment (Laskemoen et al. 2020). Importantly, this study further investigated the mediating role of insomnia in the link between a history of childhood maltreatment and mental health in adulthood and discovered that insomnia mediated the link between a history of childhood maltreatment and severe anxiety and depressive symptoms in adulthood (Laskemoen et al. 2020). Given the finding that insomnia significantly mediated the relationship between childhood experiences of maltreatment and severe mental health problems in adulthood, it is possible that adequate hours of sleep are significantly associated with a history of childhood maltreatment and mental health problems.

Specifically, Buysse (2014) describes that sleep health is multidimensional, which has five indicators of sleep health: (1) Sleep duration; (2) Sleep continuity/efficiency; (3) Timing; (4) Alertness/sleepiness; (5) Satisfaction/Quality. Of the five indicators, sleep duration - hours of sleep—is defined as the total amount of hours of sleep per $24 \mathrm{~h}$ (Buysse 2014). Although it has been indicated that adequate hours of sleep vary according to age, it is recommended that young adults and adults have 7 to $9 \mathrm{~h}$ of sleep on average to maintain sleep health (Hirshkowitz et al. 2015). Since adequate hours of sleep have been identified as a fundamental factor for sleep health, which is also positively associated with mental health (Consensus Conference Panel et al. 2015), it can be argued that having adequate hours of sleep can contribute to reducing the risk for mental health problems.

\section{Multiple Types of Child Maltreatment, Mental Health Problems, and Sleep Problems}

The relationships between childhood experiences of multiple types of violence and mental health outcomes have been widely investigated (Cloitre et al. 2009; Finkelhor et al. 2007; Turner et al. 2010). Specifically, Finkelhor and colleagues (2007) found that children with experiences of multiple types of maltreatment had higher risk for having depression, anxiety, aggression, and anger than did children with experiences of a single type of violence. Another study also indicated that children and youth who experienced multiple types of child maltreatment had a greater risk of anger, anxiety, and depression, compared to those who experienced the recurrence of the same type of child maltreatment (Turner et al. 2010). Moreover, Cloitre et al. (2009) discovered that adults who experienced multiple types of child maltreatment as a child were at high risk for PTSD symptoms and emotion dysregulation as an adult.

While significant associations between experiences of multiple types of child maltreatment and mental health problems have been widely identified (Cloitre et al. 2009; Finkelhor et al. 2007; Turner et al. 2010), the associations between experiences of multiple types of child maltreatment and sleep problems appear to vary by the severity and types 
of child maltreatment (Bader et al. 2007). Specifically, one study examining the association between the severity of childhood maltreatment and insomnia found that insomnia was prevalent among adults who had experiences of severe child maltreatment during childhood (Bader et al. 2007). In particular, those with experiences of severe emotional and physical neglect were shown to have more sleep problems (Bader et al. 2007). In contrast, another study revealed that adults who experienced multiple types of abuse (i.e. physical, emotional, and sexual abuse) had greater risk for sleep problems even if they experienced physical and sexual abuse infrequently (Greenfield et al. 2011). Given the mixed results, the current study focused on multiple types of child maltreatment and explored the relationships between multiple types of child maltreatment, sleep problems, and anxiety in former foster youth and low-income young adults.

In addition, previous sleep studies have primarily used self-report measures to assess sleep problems, which might possibly lead to biased results. Specifically, studies examining the relationships between trauma histories and sleep problems have shown that there are significant discrepancies between self-report measures of sleep and objective sleep measures (e.g., actigraphy) (Caldwell and Redeker 2005; Ghadami et al. 2015; Girschik et al. 2012; Slightam et al. 2018). For example, a review study of sleep disturbances in people with PTSD indicated that those with PTSD were likely to assess their sleep quality as poorer than sleep quality assessed by objective measures (Caldwell and Redker 2005). Consistent with this finding, another study of veterans with chronic PTSD also found that the veterans with PTSD were likely to report fewer hours of sleep and a lower sleep efficiency than were measured by the actigraphy, resulting in over-reported sleep problems by the study participants (Ghadami et al. 2015). Given the reporting bias, it can possibly be assumed that those with trauma histories are more likely to perceive their sleep health as poor because of their traumatic stress. Thus, it is important to use objective measures when assessing hours of sleep among populations, particularly those with trauma histories, to reduce the risk of bias.

\section{The Current Study}

Although the previous studies have documented significant relationships between a history of childhood maltreatment and sleep problems and between a history of childhood of maltreatment and mental health problems, little is known about the relationships between experiences of multiple types of childhood maltreatment, sleep problems, and anxiety in former foster youth and low-income young adults. To fill the research gap, the current study explored whether experiences of multiple types of childhood maltreatment would be associated with anxiety in low-income young adults and former foster youth. Secondly, the current study investigated whether a history of foster care placement would be associated with anxiety, even after controlling for hours of sleep. Lastly, this study sought to identify the relationships of multiple types of childhood maltreatment to anxiety after controlling for hours of sleep, a history of foster care placement, lifetime trauma, and demographic information. Of the particular interest is the change in the relationship between experiences of multiple types of child maltreatment and anxiety, after including the hours of sleep variable as a control variable. Identification of the associations between experiences of multiple types of child maltreatment, hours of sleep, and anxiety in the low-income young adults and former foster youth can guide social workers or service providers to develop intervention and prevention strategies that are more effective for reducing the risk for anxiety.

Research questions in the current study were as follows:

(1) Is experiencing multiple types of childhood maltreatment associated with anxiety in low-income young adults and former foster youth?

(2) Is a history of foster care placement related to anxiety after controlling for hours of sleep?

(3) Is experiencing multiple types of childhood maltreatment associated with anxiety after controlling for hours of sleep, a history of foster care placement, lifetime trauma, and demographic information?

\section{Method}

\section{Study Design}

This cross-sectional study used data collected from a survey conducted from September 2016 to March 2018. A total sample was 143 low-income young adults aged 18 to 24. Of the total, 92 had spent time in foster care, and 51 young adults had never been in foster care. The data were collected from these two groups at the same time. There were no missing data on the measures of study variables. All activities were approved by the Institutional Review Board at [BLIND].

\section{Participants}

The study sample $(\mathrm{N}=143)$ was recruited from two agencies that provided employment services for low-income youth in a northeastern city in the United States. Fliers were posted in common areas and interested people contacted the study team. Youth were then informed about the study and those who agreed to participate signed informed 
consent forms. Participants completed a self-administered questionnaire assessing their history of childhood maltreatment, lifetime trauma, hours of sleep, anxiety, etc. All study participants received a wearable device, FitBit, to measure hours of sleep for four consecutive nights. A power analysis was performed to identify whether the total sample size was met for multivariate regression analyses. Based on Cohen's (1998) conventions, the power analysis showed that at least 82 participants were needed as a total sample size, with the medium effect size and a power of 0.80 , to show a statistically significant increase in $R^{2}$. The study sample size was adequate in this regard.

\section{Measures}

\section{Multiple Types of Childhood Maltreatment}

Childhood Trauma Questionnaire (CTQ; Bernstein et al. 1998), which is a self-report measure assessing experiences of multiple types of childhood maltreatment, was used. In the current study, three items were drawn from the CTQ to measure physical abuse, sexual abuse, and neglect. It was coded 1 if the respondent answered "Yes" to a question "I believe I was physically abused" and coded 0 for "No." Since the current study focused on measuring experiences of multiple types of child maltreatment instead of the severity or frequency of child maltreatment, each response was summed to calculate multiple types of maltreatment. A total score ranged from 0 (no abuse and neglect) to 3 (exposed to physical abuse, sexual abuse, and neglect). The CTQ measure has moderate to high internal consistency $(\alpha=0.79-0.95)$, and a good test-retest reliability (correlation coefficient ranged from 0.81 to 0.91 ) (Gardner et al. 2014).

\section{Lifetime Trauma}

The Brief Trauma Questionnaire (BTQ; Schnurr et al. 1999) is a 10-item self-report questionnaire. The measure evaluates 10 potentially traumatic life events including death of a close friend or family member, natural disasters, and life-threatening illnesses. Kappa coefficients for all event-specific items range from 0.74 to 1.00 (Schnurr et al. 2002). Tests of interrater reliability has been shown to be very good for and criterion validity has been demonstrated repeatedly with strong associations found between PTSD symptomology and higher BTQ scores (Harville et al. 2015). "Yes" scores were coded as 1 and "no" was coded as 0 . Overall scores ranged from 0 to 10 .

\section{Anxiety}

The Beck Anxiety Inventory (BAI; Beck et al. 1988) is a 21-item self-report questionnaire measuring anxiety levels. Items are scored on a 4-point Likert scale ranging from 0 (not at all) to 3 (severely; it bothered me a lot). Each item was summed to calculate anxiety level with a possible maximum score of 63 . The internal consistency for the BAI in the prior literature was high (Cronbach's $\alpha=0.92$ ), and the test-retest reliability was 0.75 (Beck et al. 1988). The measure showed moderate concurrent validity $(0.51)$ with the Hamilton Anxiety Rating Scale and marginal convergent validity with the Hamilton Depression Rating Scale (0.25) (Beck et al. 1988).

\section{Hours of Sleep}

Hours of sleep were measured with a FitBit Charge, which was worn $24 \mathrm{~h}$ a day for four consecutive nights. The FitBit Charge collects a wide range of data, including total time asleep, length of time to fall asleep (sleep onset latency), and number of nighttime awakenings. These aspects are all considered to be important quantitative measures of sleep problems (Buysse et al. 2006). While the highest standard of sleep measurement is polysomnography, the FitBit Charge has demonstrated effectiveness in measuring sleep time (Brooke et al. 2017) and is more affordable, more comfortable, and requires less of a time commitment from participants than spending nights in a sleep lab. FitBits have been determined to be comparable to polysomnography in estimating total sleep time and are highly correlated with more expensive monitors used in medical research in measuring sleep efficiency (Mantua et al. 2016). FitBits also show strong inter-device reliability (Montgomery-Downs, Insana and Bond 2012). All FitBit data were downloaded for analysis. There were no missing data.

\section{History of Foster Care Placement}

The study participants were asked if they had been in foster care. The dichotomous response was coded 1 if the respondents answered "Yes" and coded 0 if "No."

\section{Data Analysis}

All analyses were conducted using STATA V.16. First, univariate analysis was conducted to show sample characteristics and descriptive statistics of the key study variables. Bivariate analysis was then performed to identify whether the key study variables were significantly correlated. Multiple regression analyses using a hierarchical regression model were performed because the primary aim of the study was to explore whether there would be significant associations 
between multiple types of child maltreatment and anxiety, even after controlling for hours of sleep, a history of foster care placement, a lifetime history of trauma, and demographic information. Additional independent variables were added to the hierarchical regression models to identify a unique and significant contribution to explaining the variance of anxiety, controlling for other independent variables. While sex and race variables were controlled, age variable was not controlled because it did not significantly vary in our study sample. The multicollinearity diagnostic test presented the tolerance and the VIF of each key independent variable, $\mathrm{VIF}=2.64$ for the multiple types of maltreatment variables; $\mathrm{VIF}=2.32$ for the hours of sleep variable; $\mathrm{VIF}=2.20$ for the lifetime trauma variable, and VIF $=1.12$ for the foster care variable. The diagnostic test indicated that there was no multicollinearity concern with these independent variables to perform the multiple regression analyses.

\section{Results}

\section{Descriptive Statistics}

Table 1 summarizes the descriptive statistics of the key study variables. It was found that the number of types of child maltreatment ranged from 0 (perceived as not being victimized) to 3 (perceived as being exposed to child physical and sexual abuse and neglect). The number of types of child maltreatment variable adjustably had a normal distribution (skewness $=-0.22$; kurtosis $=2.02$ ). On average, the study participants experienced at least more than one type of child maltreatment $(M=1.44, S D=0.95)$.

The lifetime histories of trauma ranged from 0 to 8 , and the distribution was skewed to the right (skewness $=0.56$; kurtosis $=1.98$ ). The average number of experiences of traumatic events among the study sample was 2.48 , indicating that the sample experienced two lifetime traumatic events on average. Moreover, it was shown that hours of sleep ranged from $4 \mathrm{~h}$ to $9.5 \mathrm{~h}$ (skewness $=1.1$; kurtosis $=4.01$ ). The mean hours of sleep of the study sample were about 5.61. The study sample sleep less than the recommended hours of sleep for young adults and adults (7-9 h) on average (Centers for Disease Control and Prevention 2017). The levels of anxiety among the study sample ranged from 1 to 45 (skewness $=0.64$; kurtosis $=2.72$ ). The average level of anxiety in our study's total sample was $16.28(S D=9.26)$, reflecting mild to moderate anxiety according to the Beck Anxiety Inventory (Morin et al., 1999). The mean level of anxiety of the former foster youth was significantly higher $(M=17.98, S D=9.22)$ than that of the low-income young adults $(M=13.22, S D=8.61), t(141)=-3.03, p=0.003$.

\section{Multiple Regression Models}

The assumptions for the multiple regression analyses were tested to perform the multiple regression analyses. The dependent variable — anxiety — had linear relationships with each independent variable (i.e., the number of types of child
Table 1 Descriptive statistics for study variables $(\mathrm{N}=143)$

\begin{tabular}{|c|c|c|c|c|c|c|c|c|}
\hline Variables & $N$ & $\%$ & $M$ & $S D$ & $V$ & Range & Skew & Kurt \\
\hline Anxiety & - & - & 16.28 & 9.26 & 85.84 & $1-45$ & 0.64 & 2.72 \\
\hline Types of childhood maltreatment & - & - & 1.44 & 0.95 & 0.9 & $0-3$ & -0.23 & 2.03 \\
\hline Lifetime trauma & - & - & 2.48 & 2.4 & 5.76 & $0-8$ & 0.56 & 1.98 \\
\hline Hours of sleep & - & - & 5.61 & 1.25 & 1.56 & $4-9.5$ & 1.1 & 4.01 \\
\hline \multicolumn{9}{|l|}{ Foster care } \\
\hline No & 51 & 35.66 & - & - & - & - & - & - \\
\hline Yes & 92 & 64.34 & - & - & - & - & - & - \\
\hline \multicolumn{9}{|l|}{ Childhood maltreatment } \\
\hline No & 31 & 21.68 & - & - & - & - & - & - \\
\hline One type & 33 & 23.08 & - & - & - & - & - & - \\
\hline Two types & 64 & 44.76 & - & - & - & - & - & - \\
\hline Three types & 15 & 10.49 & - & - & - & - & - & - \\
\hline \multicolumn{9}{|l|}{ Sex } \\
\hline Males & 67 & 46.85 & - & - & - & - & - & - \\
\hline Females & 76 & 53.15 & - & - & - & - & - & - \\
\hline \multicolumn{9}{|l|}{ Race } \\
\hline White & 34 & 23.78 & - & - & - & - & - & - \\
\hline $\begin{array}{l}\text { African } \\
\text { American }\end{array}$ & 99 & 69.23 & - & - & - & - & - & - \\
\hline Multiracial & 10 & 6.99 & - & - & - & - & - & - \\
\hline
\end{tabular}


maltreatment, lifetime trauma, and hours of sleep); these linear relationships were essential before conducting the multiple regression analyses. In terms of homoscedasticity, the scatterplot of studentized residuals against values of the independent variables showed that the spread of residuals did not appear to have a perfect homoscedasticity. Yet the kernel density plot indicated that the unstandardized residuals seemed to be smoothed, meeting the assumption of normality ( performed after the assumptions of linearity and normality were met.

Table 2 displays five hierarchal regression models, and each model identified the extent to which added independent variables explained variances in anxiety. At first, the multiple regression analysis of anxiety on the control variables (i.e., sex, race) was conducted, and the first model was not significant. In the second model, the foster care variable was added to examine whether the levels of anxiety would be accounted for by a history of foster care placement. The second model was significant, $[F(4,138)=2.67, p<0.05]$, although the foster care variable explained only $7 \%$ of variance in anxiety. On average, the former foster youth had 4.62 points higher levels of anxiety than did other young adults who had never been in foster care, $(t=2.90, p<0.05)$, controlling for sex and race.

The number of types of child maltreatment variable was added to the third model, and this model was significant, $[F$ $(7,135)=16.89, p<0.001]$, with $R^{2}=0.47$. The substantial $R^{2}$ change, accounting for an additional $40 \%$ of the variance in anxiety, was significant, controlling for other variables, i.e., sex, race, a history of foster care placement. In the third model, the number of types of child maltreatment was found to be strongly associated with anxiety. The number of types of child maltreatment was positively related to the levels of anxiety. Specifically, the study participants who experienced a single type of child maltreatment had 3 points higher levels of anxiety than did those who had no experiences of childhood maltreatment. More importantly, the level of anxiety was 12 points higher for those who experienced two different types of child maltreatment and was almost 18 points higher for those who experienced three different types of child maltreatment, compared to those who had no experience of child maltreatment, controlling for other variables.

The fourth multiple regression analysis was performed to examine the extent to which a lifetime history of trauma would explain the variance in anxiety. The model was significant, $[F(8,134)=20.21, p<0.001]$, and all of these predictors accounted for $55 \%$ of variance in anxiety. A history of foster care placement remained associated with anxiety, and a one-unit change in the number of lifetime traumatic events increased approximately 1.57 points of the levels of anxiety $(t=4.86, p<0.001)$ while controlling for other variables. In other words, a lifetime history of trauma uniquely contributed to explaining the variance in anxiety among the study participants. An additional $8 \%$ of the $R^{2}$ was statistically significant. However, it should be noted that the level of anxiety among those without childhood experiences of maltreatment was not significantly different from that of those who experienced a single type of childhood maltreatment. In contrast, the levels of anxiety were significantly higher in groups who experienced two and three different types of child maltreatment, compared to those without childhood experiences of maltreatment.

In the final model, the hours of sleep variable was added to examine whether anxiety would be accounted for by hours of sleep and whether the relationship between experiences of multiple types of child maltreatment and anxiety would change, after controlling for hours of sleep. The final model was significant, $[F(9133)=29.88, p<0.001]$, with $R^{2}=0.67$. Compared to the fourth model, the $R^{2}$ increased by approximately $12 \%$, and the increase was significant. A one-unit increase in hours of sleep decreased approximately 4.11 points of levels of anxiety $(t=-7.01, p<0.001)$, controlling for other variables. Surprisingly, a lifetime history of trauma and hours of sleep remained significantly associated with anxiety, even after all other independent variables were controlled for. In contrast, neither experiences of multiple types of child maltreatment nor a history of foster care placement accounted for variance in anxiety after including hours of sleep as a control variable.

\section{Discussion}

The results of the study provide additional data to support the prior evidence on the relationships between childhood experiences of maltreatment and poor mental health outcomes among low-income young adults and former foster youth (Bruskas and Tessin 2013). In particular, this study focused on examining the extent to which multiple types of childhood maltreatment account for the variance in anxiety among low-income young adults and former foster youth who are at high risk for sleep problems and mental health problems. In addition, the study investigated whether a history of placement in foster care would be associated with anxiety, even after controlling for hours of sleep. Moreover, the study examined whether there would be a change in the associations between multiple types of child maltreatment and anxiety, after controlling for other independent variables, including the hours of sleep variable.

In consistent with the previous evidence on the high prevalence of mental health problems among those with a history of placement in foster care (Pecora et al. 2009; Shin 2005), the current study indicated that a history of foster care placement was significantly associated with an increased level of anxiety. Additionally, this study revealed that experiences 


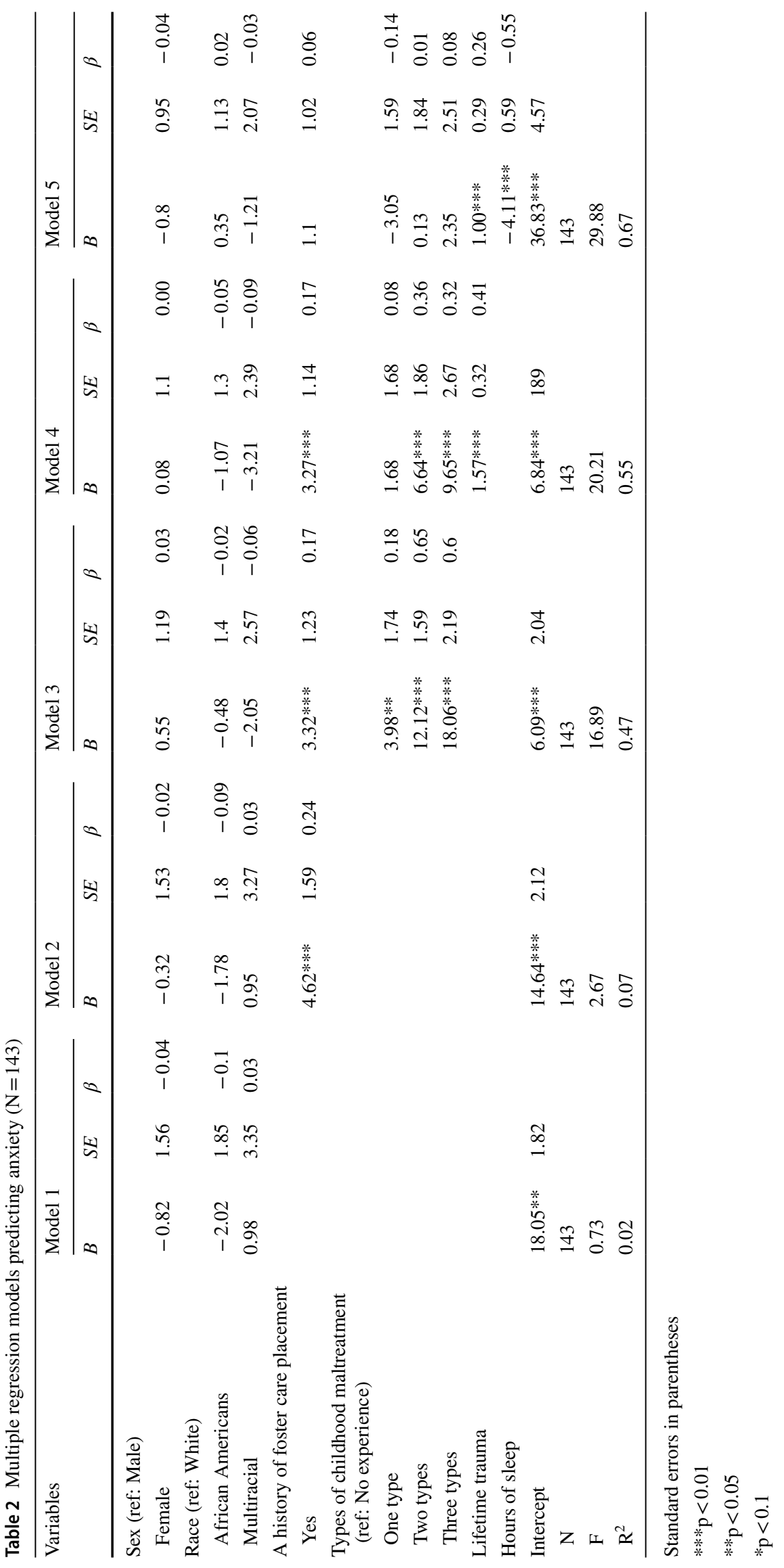


of multiple types of childhood maltreatment were positively associated with anxiety in low-income young adults and former foster youth. Particularly, the experiences of multiple types of childhood maltreatment remained significantly associated with anxiety after a history of foster care placement and demographic information were taken into account. This evidence adds to the previous findings that experiences of multiple types of childhood maltreatment are associated with an elevated risk for mental health problems, including anxiety (Finkelhor et al. 2007), It is worth noting that while experiences of more than two different types of child maltreatment were significantly associated with anxiety, experiences of a single type of child maltreatment were not related to anxiety after controlling for a lifetime history of trauma. This result also adds additional supports to the previous findings that the experiences of multiple types of child maltreatment significantly differ from the experiences of a single type of child maltreatment (Edwards et al. 2003; Turner et al. 2010). More importantly, this study discovered that both experiences of multiple types of child maltreatment and a history of placement in foster care were no longer related to anxiety after including hours of sleep as a control variable. It can be elicited from these findings that adequate hours of sleep may potentially play a role in buffering anxiety among low-income young adults even if they had a history of foster care placement or experienced multiple types of child maltreatment. However, it should be noted that a lifetime history of trauma remained significantly associated with anxiety, even after controlling for hours of sleep and other independent variables. Although the current study found that the total sample had mild to moderate anxiety (Morin et al. 1999), the former foster youth had a higher mean score of anxiety than did the low-income young adults, supporting the prior evidence on the high prevalence of anxiety among former foster youth (Pecora et al. 2009). This research evidence suggests that it is critical for former foster youth and low-income young adults to receive mental health services in order to alleviate anxiety.

Getting help for mental health problems, however, often involves encountering challenging barriers. In spite of recent efforts to combat it, mental illnesses of all forms carry a heavy burden of stigma. A recent systematic review of anxiety disorders and stigma by Curcio and Corboy (2020) showed that younger people showed the highest stigmatizing attitudes and actions, and this negatively impacts treatment seeking. Poor sleep, however, does not carry such a burden of stigma. Most people are more comfortable talking about sleep problems than they are talking about their anxiety symptoms. Such lack of stigma around poor sleep means interventions to improve sleep could be an effective way to target young adults who may not seek mental health treatment. Indeed, a large body of research testing the effectiveness of computerized CBT for insomnia has widely identified that the computerized CBT is effective not only for alleviating sleep problems and mental health problems but for reducing stigma in the utilization of mental health services (Andrews et al. 2010; Cheng and Dizon 2012; Ivarsson et al. 2014). However, since these intervention studies did not include former foster youth and low-income young adults as a target sample, more future research needs to test whether sleep-focused interventions or computerized CBT for insomnia would be effective in reducing the risk of anxiety for former foster youth and low-income young adults who experienced multiple types of childhood maltreatment.

\section{Limitations}

The results of the study should be interpreted in light of methodological limitations. First, the majority of the study participants were African Americans (White $=24 \%$; African Americans $=64 \%$; multiracial $=7 \%$ ), so the study results would differ if the study included more samples from diverse racial/ethnic backgrounds. Second, since this study used self-report measures to assess the study participants' childhood experiences of maltreatment, a lifetime history of trauma, and anxiety, there might be response bias or social desirability bias. Lastly, this study investigated only a unidirectional association between hours of sleep and anxiety. While some studies provide evidence that sleep problems predict adverse mental health outcomes (Gregory and O'Connor 2002), there is growing evidence to support for the bidirectional relationship between sleep problems and mental health problems (Shanahan et al. 2014; Zou et al. 2020). Since this study used cross sectional data and focused on the unidirectional relationship between hours of sleep and anxiety, it is not possible to conclude that fewer hours of sleep cause anxiety. In addition, the study did not collect data of hours of sleep and anxiety at multiple time points. As such, it is possible to assume that anxiety could lead to fewer hours of sleep or that there would be a bidirectional link between fewer hours of sleep and anxiety. Therefore, more long-term design studies are needed to test the bidirectionality of inadequate hours of sleep and anxiety among former foster youth and low-income young adults.

\section{Implications}

Despite the limitations of the study, the results provide important research and clinical implications. Specifically, in terms of measures for hours of sleep, the current study used an objective measure for hours of sleep (FitBits), which can compensate for the limitations of self-report measures for hours of sleep as well as high cost of polysomnography (Martin and Hakim 2011). In addition, these findings 
can guide mental health professionals or social workers to develop and implement effective treatments when working with former foster youth or young adults who experienced multiple types of childhood maltreatment and other lifetime traumatic events. Specifically, providing treatments targeted at addressing insufficient hours of sleep can also be effective for mitigating anxiety since multiple types of child maltreatment and a history of foster care placement were no longer related to anxiety after controlling for hours of sleep. However, since a lifetime history of trauma remained significantly associated with anxiety even after controlling for hours of sleep, it seems critical to provide them with treatments targeted at addressing both traumatic stress and inadequate hours of sleep. Future studies need to conduct more interventions to test whether these interventions are effective in mitigating anxiety.

\section{Conclusions}

Childhood experiences of multiple types of maltreatment have been positively associated with fewer hours of sleep and higher levels of anxiety in low-income young adults and former foster youth. Noticeably, childhood experiences of multiple types of maltreatment were no longer related to increased levels of anxiety after controlling for hours of sleep. This finding suggests that having adequate amounts of sleep may possibly play a role in mitigating the negative impacts of childhood experiences of multiple types of maltreatment on anxiety although the buffering role of sleep in this relationship should be tested in long-term design studies. Additionally, in terms of clinical interventions, it is critical for social workers and service providers to screen for the high-risk populations who have experiences of multiple types of childhood maltreatment, sleep problems, and anxiety. More importantly, social workers and service providers need to provide low-income young adults and former foster youth with interventions aimed at addressing both sleep problems and traumatic stress, which may in turn reduce the risk for anxiety.

\section{References}

Ai, A. L., Foster, L. J. J., Pecora, P. J., Delaney, N., \& Rodriguez, W. (2013). Reshaping child welfare's response to trauma: Assessment, evidence-based intervention, and new research perspectives. Research on Social Work Practice, 23(6), 651-668.

Andrews, G., Cuijpers, P., Craske, M. G., McEvoy, P., \& Titov, N. (2010). Computer therapy for the anxiety and depressive disorders is effective, acceptable and practical health care: a meta-analysis. PLOS ONE, 5(10), e13196.

Bader, K., Schaefer, V., Schenkel, M., Nissen, L., \& Schwander, J. (2007). Adverse childhood experiences associated with sleep in primary insomnia. Journal of Sleep Research, 16(3), 285-296.
Bass, S., Shields, M. K., \& Behrman, R. E. (2004). Children, Families, and Foster Care: Analysis and Recommendations. The Future of Children, 14(1), 4. https://doi.org/10.2307/1602752.

Beck, A. T., Epstein, N., Brown, G., \& Steer, R. A. (1988). An inventory for measuring clinical anxiety: Psychometric properties. Journal of Consulting and Clinical Psychology, 56(6), 893-901.

Bernstein, D. P., \& Fink, L. (1998). Childhood Trauma Questionnaire: A retrospective self-report manual. San Antonio, TX: The Psychological Cooperation.

Brindle, R. C., Cribbet, M. R., Samuelsson, L. B., Gao, C., Frank, E., Krafty, R. T., \& Hall, M. H. (2018). The relationship between childhood trauma and poor sleep health in adulthood. Psychosomatic Medicine, 80(2), 200.

Brooke, S. M., An, H.-S., Kang, S.-K., Noble, J. M., Berg, K. E., \& Lee, J.-M. (2017). Concurrent Validity of Wearable Activity Trackers Under Free-Living Conditions. Journal of Strength and Conditioning Research, 31(4), 1097-1106. https://doi. org/10.1519/jsc.0000000000001571.

Bruskas, D., \& Tessin, D. H. (2013). Adverse childhood experiences and psychosocial well-being of women who were in foster care as children. The Permanente Journal, 17(3), e131.

Buysse, D. J. (2014). Sleep health: Can we define it? Does it matter? Sleep, 37(1), 9-17.

Buysse, D. J., Ancoli-Israel, S., Edinger, J. D., Lichstein, K. L., \& Morin, C. M. (2006). Recommendations for a Standard Research Assessment of Insomnia. Sleep, 29(9), 1155-1173. https://doi. org/10.1093/sleep/29.9.1155.

Caldwell, B. A., \& Redeker, N. (2005). Sleep and trauma: An overview. Issues in Mental Health Nursing, 26(7), 721-738. https:// doi.org/10.1080/01612840591008294.

Centers for Disease Control and Prevention (2017). Sleep and sleep disorders. Retrieved from https://www.cdc.gov/sleep/data_stati stics.html

Cheng, S. K., \& Dizon, J. (2012). Computerised cognitive behavioural therapy for insomnia: a systematic review and meta-analysis. Psychotherapy and Psychosomatics, 81(4), 206-216.

Cloitre, M., Stolbach, B. C., Herman, J. L., Kolk, B. V. D., Pynoos, R., Wang, J., \& Petkova, E. (2009). A developmental approach to complex PTSD: Childhood and adult cumulative trauma as predictors of symptom complexity. Journal of Traumatic Stress, 22(5), 399-408.

Cohen, J. (1988). Statistical power analysis for the behavioral sciences. L. Erlbaum Associates.

Consensus Conference Panel, Watson, N. F., Badr, M. S., Belenky, G., Bliwise, D. L., Buxton, O. M., \& Kushida, C. (2015). Recommended amount of sleep for a healthy adult: a joint consensus statement of the American Academy of Sleep Medicine and Sleep Research Society. Journal of Clinical Sleep Medicine, 11(6), 591-592.

Courtney, M. E., Lee, J., \& Perez, A. (2011). Receipt of help acquiring life skills and predictors of help receipt among current and former foster youth. Children and Youth Services Review, 33(12), 2442-2451.

Curcio, C., \& Corboy, D. (2020). Stigma and anxiety disorders: A systematic review. Stigma and Health, 5(2), 125-137. https://doi. org/10.1037/sah0000183.

Edwards, V. J., Holden, G. W., Felitti, V. J., \& Anda, R. F. (2003). Relationship Between Multiple Forms of Childhood Maltreatment and Adult Mental Health in Community Respondents: Results from the Adverse Childhood Experiences Study. American Journal of Psychiatry, 160(8), 1453-1460. https://doi.org/10.1176/appi. ajp.160.8.1453.

Farmer, E. M., Burns, B. J., Chapman, M. V., Phillips, S. D., Angold, A., \& Costello, E. J. (2001). Use of mental health services by youth in contact with social services. Social Service Review, 75(4), 605-624. 
Finkelhor, D., Ormrod, R. K., \& Turner, H. A. (2007). Poly-victimization: A neglected component in child victimization. Child Abuse \& Neglect, 31(1), 7-26. https://doi.org/10.1016/j.chiab u.2006.06.008.

Gardner, F. L., Moore, Z. E., \& Dettore, M. (2014). The Relationship Between Anger, Childhood Maltreatment, and Emotion Regulation Difficulties in Intimate Partner and Non-Intimate Partner Violent Offenders. Behavior Modification, 38(6), 779-800. https ://doi.org/10.1177/0145445514539346.

Ghadami, M. R., Khaledi-Paveh, B., Nasouri, M., \& Khazaie, H. (2015). PTSD-related paradoxical insomnia: An actigraphic study among veterans with chronic PTSD. Journal of Injury \& Violence Research, 7(2), 54-58. https://doi.org/10.5249/jivr. v7i2.607.

Girschik, J., Fritschi, L., Heyworth, J., \& Waters, F. (2012). Validation of self-reported sleep against actigraphy. Journal of Epidemiology, 22(5), 462-468. https://doi.org/10.2188/jea.JE20120012.

Glod, C. A., Teicher, M. H., Hartman, C. R., \& Harakal, T. (1997). Increased nocturnal activity and impaired sleep maintenance in abused children. Journal of the American Academy of Child \& Adolescent Psychiatry, 36(9), 1236-1243. https://doi. org/10.1097/00004583-199709000-00016.

Greenfield, E. A., Lee, C., Friedman, E. L., \& Springer, K. W. (2011). Childhood Abuse as a Risk Factor for Sleep Problems in Adulthood: Evidence from a U.S. National Study. Annals of Behavioral Medicine, 42(2), 245-256. https://doi.org/10.1007/s1216 0-011-9285-x.

Häggman-Laitila, A., Salokekkilä, P., \& Karki, S. (2019). Young People's Preparedness for Adult Life and Coping After Foster Care: A Systematic Review of Perceptions and Experiences in the Transition Period. Child \& Youth Care Forum, 48(5), 633-661. https:// doi.org/10.1007/s10566-019-09499-4.

Harville, E. W., Jacobs, M., \& Boynton-Jarrett, R. (2015). When is exposure to a natural disaster traumatic? Comparison of a trauma questionnaire and disaster exposure inventory. PLoS One, 10(4), $1-8$.

Havlicek, J. R., Garcia, A. R., \& Smith, D. C. (2013). Mental health and substance use disorders among foster youth transitioning to adulthood: Past research and future directions. Children and Youth Services Review, 35(1), 194-203. https://doi.org/10.1016/j.child youth.2012.10.003.

Hillen, T., \& Gafson, L. (2015). Why good placements matter: preplacement and placement risk factors associated with mental health disorders in pre-school children in foster care. Clinical Child Psychology and Psychiatry, 20(3), 486-499.

Hirshkowitz, M., Whiton, K., Albert, S. M., Alessi, C., Bruni, O., DonCarlos, L., \& Neubauer, D. N. (2015). National Sleep Foundation's sleep time duration recommendations: methodology and results summary. Sleep Health, 1(1), 40-43.

Ho, F. Y. Y., Chan, C. S., \& Tang, K. N. S. (2016). Cognitive-behavioral therapy for sleep disturbances in treating posttraumatic stress disorder symptoms: a meta-analysis of randomized controlled trials. Clinical Psychology Review, 43, 90-102.

Ivarsson, D., Blom, M., Hesser, H., Carlbring, P., Enderby, P., Nordberg, R., \& Andersson, G. (2014). Guided internet-delivered cognitive behavior therapy for post-traumatic stress disorder: a randomized controlled trial. Internet Interventions, 1(1), 33-40.

Jones, T. M., Nurius, P., Song, C., \& Fleming, C. M. (2018). Modeling life course pathways from adverse childhood experiences to adult mental health. Child Abuse \& Neglect, 80, 32-40.

Laskemoen, J. F., Aas, M., Vaskinn, A., Berg, A. O., Lunding, S. H., Barrett, E. A., \& Simonsen, C. (2020). Sleep disturbance mediates the link between childhood trauma and clinical outcome in severe mental disorders. Psychological Medicine. https://doi. org/10.1017/S0033291720000914.
Lawrence, C. R., Carlson, E. A., \& Egeland, B. (2006). The impact of foster care on development. Development and Psychopathology, 18(1), 57-76.

Mantua, J., Gravel, N., \& Spencer, R. (2016). Reliability of Sleep Measures from Four Personal Health Monitoring Devices Compared to Research-Based Actigraphy and Polysomnography. Sensors, 16(5), 646. https://doi.org/10.3390/s16050646.

Martin, J. L., \& Hakim, A. D. (2011). Wrist actigraphy. Chest, 139(6), $1514-1527$.

McMillen, J. C., Zima, B. T., Scott, L. D., Auslander, W. F., Munson, M. R., Ollie, M. T., \& Spitznagel, E. L. (2005). Prevalence of psychiatric disorders among older youths in the foster care system. Journal of the American Academy of Child \& Adolescent Psychiatry, 44(1), 88-95. https://doi.org/10.1097/01.chi.00001 45806.24274.d2.

Montgomery-Downs, H. E., Insana, S. P., \& Bond, J. A. (2012). Movement toward a novel activity monitoring device. Sleep \& Breathing, 16(3), 913-917. https://doi.org/10.1007/s11325-011-0585-y.

Morin, C. M., Landreville, P., Colecchi, C., McDonald, K., Stone, J., \& Ling, W. (1999). The Beck Anxiety Inventory: psychometric properties with older adults. Journal of Clinical Geropsychology, 5(1), 19-29.

Oswald, S. H., Heil, K., \& Goldbeck, L. (2009). History of Maltreatment and Mental Health Problems in Foster Children: A Review of the Literature. Journal of Pediatric Psychology, 35(5), 462472. https://doi.org/10.1093/jpepsy/jsp114.

Pecora, P. J., White, C. R., Jackson, L. J., \& Wiggins, T. (2009). Mental health of current and former recipients of foster care: a review of recent studies in the USA. Child \& Family Social Work, 14(2), 132-146. https://doi.org/10.1111/j.1365-2206.2009.00618.x.

Perepletchikova, F., Ansell, E., \& Axelrod, S. (2012). Borderline Personality Disorder Features and History of Childhood Maltreatment in Mothers Involved with Child Protective Services. Child Maltreatment, 17(2), 182-190. https://doi.org/10.1177/10775 59512448471.

Pillai, V., Anderson, J. R., Cheng, P., Bazan, L., Bostock, S., Espie, C. A., \& Drake, C. L. (2015). The anxiolytic effects of cognitive behavior therapy for insomnia: preliminary results from a webdelivered protocol. Journal of Sleep Medicine and Disorders, 2(2).

Pilowsky, D. J., \& Wu, L. T. (2006). Psychiatric symptoms and substance use disorders in a nationally representative sample of American adolescents involved with foster care. Journal of Adolescent Health, 38(4), 351-358.

Rubin, D. M., O'Reilly, A. L., Luan, X., \& Localio, A. R. (2007). The impact of placement stability on behavioral well-being for children in foster care. Pediatrics, 119(2), 336-344.

Salazar, A. M., Keller, T. E., \& Courtney, M. E. (2011). Understanding social support's role in the relationship between maltreatment and depression in youth with foster care experience. Child Maltreatment, 16(2), 102-113.

Schnurr, P. P., Spiro, A., Vielhauer, M. J., Findler, M. N., \& Hamblen, J. L. (2002). Trauma in the lives of older men: Findings from the Normative Aging Study. Journal of Clinical Geropsychology, 8(3), 175-187.

Schnurr, P., Vielhauer, M., Weathers, F., \& Findler, M. (1999). The brief trauma questionnaire. White River Junction, VT: National Center for PTSD.

Shanahan, L., Copeland, W. E., Angold, A., Bondy, C. L., \& Costello, E. J. (2014). Sleep problems predict and are predicted by generalized anxiety/depression and oppositional defiant disorder. Journal of the American Academy of Child \& Adolescent Psychiatry, 53(5), 550-558.

Shin, S. H. (2005). Need for and actual use of mental health service by adolescents in the child welfare system. Children and Youth Services Review, 27(10), 1071-1083. https://doi.org/10.1016/j. childyouth.2004.12.027. 
Slightam, C., Petrowski, K., Jamison, A. L., Keller, M., Bertram, F., Kim, S., \& Roth, W. T. (2018). Assessing sleep quality using selfreport and actigraphy in PTSD. Journal of Sleep Research, 27(3), e12632. https://doi.org/10.1111/jsr.12632.

Swire, M. R., \& Kavaler, F. (1977). The health status of foster children. Child Welfare, 56(10), 635-653.

Szilagyi, M. (2012). The pediatric role in the care of children in foster and kinship care. Pediatrics in Review, 33(11), 496.

Turner, H. A., Finkelhor, D., \& Ormrod, R. (2010). Poly-victimization in a national sample of children and youth. American Journal of Preventive Medicine, 38(3), 323-330.

U.S. Department of Health \& Human Services, Administration for Children and Families, Administration on Children, Youth and Families, Children's Bureau. (2020). Child Maltreatment 2018. Available from https://www.acf.hhs.gov/cb/research-data-techn ology/statistics-research/child-maltreatment.

Villagrana, M., Guillen, C., Macedo, V., \& Lee, S. Y. (2018). Perceived self-stigma in the utilization of mental health services in foster care and post foster care among foster care alumni. Children and Youth Services Review, 85, 26-34.
Werner, E. E. (1989). High-risk children in young adulthood: A longitudinal study from birth to 32 years. American Journal of Orthopsychiatry, 59(1), 72-81. https://doi.org/10.1111/j.1939-0025.1989. tb01636.x.

Widom, C. S. (1999). Posttraumatic stress disorder in abused and neglected children grown up. American Journal of Psychiatry, 156(8), 1223-1229. https://doi.org/10.1176/ajp.156.8.1223.

Zou, P., Wang, X., Sun, L., Liu, K., Hou, G., Yang, W., \& Chen, Q. (2020). Poorer sleep quality correlated with mental health problems in college students: A longitudinal observational study among 686 males. Journal of Psychosomatic Research, 136, 110177.

Publisher's Note Springer Nature remains neutral with regard to jurisdictional claims in published maps and institutional affiliations. 